\title{
Aprendizagem Corporativa em Teste de Software: Uma Revisão Sistemática da Literatura
}

\author{
Dianne Dias Silva ${ }^{1}$, Deller James Ferreira ${ }^{2}$ \\ ${ }^{1}$ Instituto de Informática - Universidade Federal de Goiás (UFG) \\ Caixa Postal 131 - 74001-970 - Goiânia - GO - Brasil \\ \{diannesilva, deller\}@inf.ufg.br
}

\begin{abstract}
Software products developed by Brazilian organizations have stood out in the global IT market. Due to the existing flaws, these companies need to improve the quality of their processes and their work techniques. In this sense, education tries to find out what directions should be taken and what are the best ways to enable an organization to establish an organizational test framework. The use of hackathons is a growing trend to promote engagement among the team during problem solving. Thus, hackathon provides an excellent framework for corporate learning in software testing to be developed in an effective and inclusive way.
\end{abstract}

Resumo. Os produtos de software desenvolvidos pelas organizações brasileiras têm se destacado no mercado mundial de TI. Devido as falhas existentes, há a necessidade dessas empresas melhorarem a qualidade dos seus processos e as suas técnicas de trabalho. Nesse sentido, a educação tenta descobrir quais rumos devem ser tomados e quais as melhores formas de capacitar uma organização para estabelecer uma estrutura organizacional de teste. $O$ uso de hackathons são uma tendência crescente para promover o engajamento entre a equipe durante resolução de problemas. Dessa forma, o hackathon oferece uma excelente estrutura para que a aprendizagem corporativa em teste de software seja desenvolvida de maneira efetiva e inclusiva.

\section{Introdução}

Nas últimas décadas a crescente demanda e a complexidade dos produtos de software, cada vez mais têm exigido que as equipes envolvidas nos projetos de desenvolvimento desses produtos sejam maiores e mais especializadas. Esse cenário, tanto do ponto de vista do processo de produção como do ponto de vista dos produtos gerados tem provocado um anseio por qualidade e produtividade [Bartié 2002]. Diante disso, as empresas de desenvolvimento de software, têm se preocupado e investido na garantia da qualidade, que está relacionada diretamente com a qualidade do processo de teste ao qual foi submetido [Staab 2003].

Ainda que haja inúmeras técnicas, políticas e metodologias na área de teste de software, existem deficiências evidentes em relação à integração entre o processo de teste e ferramentas no âmbito dessas organizações. Nesse sentido, a educação tenta adequar-se a esse progresso buscando descobrir quais rumos devem ser tomados e quais as melhores formas de preparar uma empresa para instaurar uma estrutura organizacional de teste [Meireles and Bonifácio 2015]. 
VIII Congresso Brasileiro de Informática na Educação (CBIE 2019)

Anais do XXV Workshop de Informática na Escola (WIE 2019)

Apesar dos esforços, até o momento, ainda não há uma forma de proporcionar a aprendizagem corporativa em teste de software capaz de disseminar e preservar o conhecimento adquirido ao longo do processo de desenvolvimento de software. Além disso, nenhuma pesquisa foi encontrada no contexto da aprendizagem corporativa em teste de software. Nem tampouco há revisões sistemáticas sobre o assunto, motivando a realização de uma Revisão Sistemática da Literatura (RSL) sobre a aprendizagem corporativa em teste de software. Esta revisão objetiva resumir os vários trabalhos anteriores, sintetizando essas contribuições de maneira organizada e evidenciando as lacunas na literatura.

Este artigo está organizado em cinco seções. A Seção 2, apresenta o método seguido nesta pesquisa. Na Seção 3, são demonstrados os resultados obtidos neste estudo. A Seção 4, aborda a discussão dos resultados desta pesquisa. Por fim, na Seção 5 é abrangida a conclusão assim como, os possíveis desdobramentos para trabalhos futuros.

\section{Método de Pesquisa}

A revisão da literatura foi conduzida por meio de uma abordagem sistemática proposta por Kitchenham et. al. [Kitchenham and Charter 2007] e consiste em cinco etapas sequenciais estabelecidas por Okoli [Okoli and Schabram 2010]. As questões de pesquisa foram respondidas em dois passos: especificação do objetivo da revisão da literatura e definição do protocolo de trabalho.

Em seguida, foram formuladas duas questões de pesquisa: "QP1 - Existem trabalhos que disseminam o conhecimento dos conceitos e aplicações do teste de software em uma organização?" e "QP2 - Quais as estratégias educacionais são abordadas na aprendizagem corporativa?". Nesta pesquisa, não foi encontrado nenhum estudo que aborde especificamente a aprendizagem corporativa em teste de software.

O protocolo desta RSL foi construído com base em cinco elementos do PIPOC [Kitchenham and Charter 2007]: as equipes de teste de software, as atividades de teste de software, a quantidade de defeitos detectados no ambiente de produção, a qualidade e cobertura dos casos de teste e a quantidade de defeitos detectados e a estruturação do teste de software em uma organização e, nas seguintes palavras-chave: "corporate learning in software testing", "organization learning in software testing", "training in software testing" e "education in software testing".

Após a execução desse protocolo nas bases Academic Search Premier, ACM, Elsevier, IEEE, Scopus e Springer, foi encontrado apenas 1 artigo que satisfizesse os critérios de busca. Na ausência de trabalhos direcionados especificamente para a aprendizagem em teste de software no âmbito corporativo, foi definido um novo protocolo de busca cuja execução nas bases Academic OneFile, ACM, Cambridge Core, EBSCO, Emeral Insight, Elsevier, Hindawi, Informatics in Education, JATIT, KMEL, MDKE, MECS Press, ResearchGate, SAGE, SAJIM, Scielo, Springer e Wiley através das seguintes palavras-chave: "corporate learning and software testing", "organization learning and software testing", "training and software testing" e "education and software testing" resultou em 79 artigos que atendem os critérios de busca.

Os estudos analisados neste trabalho foram filtrados através dos critérios multinível de inclusão ou exclusão, cujos processos são denominados de triagem prática e de uma avaliação da qualidade, respectivamente. A triagem prática restringiu a gama de 
VIII Congresso Brasileiro de Informática na Educação (CBIE 2019)

Anais do XXV Workshop de Informática na Escola (WIE 2019)

estudos por meio da leitura dos resumos de cada uma das pesquisas coletadas afim de garantir uma forte relação dos objetivos com as questões de pesquisa desta RSL. Esses estudos foram selecionados através de trabalhos publicados entre os anos de 2013 e 2018, do uso de palavras-chave referentes ao campo de pesquisa.

Logo após definir os idiomas, português e inglês, os protocolos foram instanciados nos mecanismos de busca acadêmicos. Após a utilização dos critérios de inclusão e exclusão foram encontrados 80 estudos. Desse total, 4 artigos tiveram que ser desconsiderados, pois foram localizados por meio das palavras-chave "training and software testing" e "education and software testing". Dessa forma, a quantidade de estudos identificados através das buscas realizadas é igual a 76.

Os seguintes critérios de inclusão foram aplicados nos protocolos de busca: "estudos escritos em Inglês ou Português", "artigos publicados nos últimos 5 anos", "trabalhos que apresentem conceitos ou estratégias educacionais de aprendizagem corporativa em teste de software", "estudos que demonstram a aplicação de conceitos ou estratégias educacionais de aprendizagem corporativa em teste de software", "artigos citados em algum trabalho referente à aprendizagem corporativa em teste de software", "trabalhos com abstract disponível" e "estudos com texto completo acessível". Como critérios de exclusão adotados, têm- se: "artigos que não abordam a aprendizagem corporativa em teste de software", "trabalhos que não respondem as questões de pesquisa" e "estudos com menos de oito páginas".

Para estabelecer os critérios de qualidade que compõem a avaliação da qualidade, houve a necessidade de adotar uma técnica de pontuação para verificar a relevância, integridade e credibilidade dos estudos selecionados. Essa avaliação ocorreu através de seis critérios de qualidade, que originaram as perguntas da avaliação: "CQ1 - Algum dos estudos transmite o conhecimento dos conceitos e aplicações de teste de software no contexto organizacional?", "CQ2 - Existem trabalhos envolvendo a aprendizagem corporativa?", "CQ3 - Foram identificados artigos referentes ao aprendizado em teste de software?", "CQ4 - As estratégias educacionais contribuem com o aprendizado organizacional?", "CQ05 - Ocorre maior integração entre as equipes de trabalho de departamentos distintos?" e "Ocorre maior integração entre as equipes de trabalho de departamentos distintos?".

A execução do método de pesquisa deste trabalho, possibilitou a extração e análise dos dados de cada um dos 76 estudos a partir dos resultados obtidos com a aplicação dos critérios de inclusão, exclusão e qualidade estabelecidos. Os artigos também foram classificados quanto a sua relevância e contribuição com o campo desta pesquisa. Os resultados da extração e análise dos dados das buscas realizadas são os estudos de Menolli et. al. [Menolli et al. 2013], de Souza et. al. [de Souza et al. 2015], Shongwue [Shongwe 2017] e Valaski et. al. [Valaski et al. 2017].

\section{Resultados}

Nenhum dos estudos analisados foi capaz de atender os objetivos e a questão de pesquisa QP1 deste trabalho. Conclui-se então que, até o momento, não se faz presente na literatura estudos que contribuem com a aprendizagem corporativa em teste de software capazes de disseminar e preservar o conhecimento obtido ao longo do processo de desenvolvimento de software. Com relação à questão QP2, foram encontrados 4 trabalhos, os quais foram 
VIII Congresso Brasileiro de Informática na Educação (CBIE 2019)

Anais do XXV Workshop de Informática na Escola (WIE 2019)

analisados para responder a questão. Esta análise também considerou a aplicabilidade dos métodos de ensino utilizados nessas pesquisas na aprendizagem de teste de software no ambiente corporativo.

Os resultados da avaliação da qualidade sobre os estudos contribuiu com as conclusões obtidas neste trabalho. Os seis critérios de qualidade foram uma garantia para que os estudos fossem selecionados, possibilitando a realização desta pesquisa. Os artigos selecionados foram publicados entre 2015 e 2018. Porém, foi possível identificar nesse período apenas 4 publicações envolvendo somente a aprendizagem corporativa.

\section{Discussão}

O principal objetivo deste estudo é apresentar as lacunas de conhecimento da aprendizagem corporativa em teste de software. Os resultados demonstram que, até o presente momento, não há nenhum estudo voltado exclusivamente para essa área de pesquisa. Entretanto, foi possível identificar através deste trabalho, 4 artigos na literatura que abrangem a Gestão do Conhecimento (GC) e a ontologia integrada como estratégias educacionais de aprendizagem no contexto organizacional.

A GC é aplicada nos estudos desenvolvidos por Menolli et. al. [Menolli et al. 2013], de Souza et. al. [de Souza et al. 2015] e Shongwe [Shongwe 2017] enquanto o trabalho Valaski et. al. [Valaski et al. 2017] adota a ontologia integrada. Apesar dos esforços, essas iniciativas demandam um alto investimento por parte das organizações, inclusive um certo nível de maturidade para que a GC ou a ontologia integrada sejam institucionalizadas. Em outras palavras, essas estratégias educacionais de aprendizagem corporativa não condizem com as peculiaridades de grande parte das organizações.

Percebendo a complexidade de aplicação de ambas e a relevância de melhorar a qualidade dos produtos de software disponibilizados no mercado, tem-se o hackathon como uma maneira de possibilitar a aprendizagem corporativa em teste de software capaz de disseminar e preservar o conhecimento obtido durante o ciclo de vida de desenvolvimento [Byrne et al. 2017].

\section{Conclusão}

Apesar das empresas de desenvolvimento de software perceberem a relevância de melhorar os seus processos e as suas técnicas de trabalho, faltam recursos e conhecimento para que essa prática seja adotada. Nesse sentido, a educação tenta descobrir quais rumos devem ser tomados e quais as melhores formas de capacitar uma organização para estabelecer uma estrutura organizacional de teste.

O principal objetivo deste trabalho foi o de evidenciar as lacunas de conhecimento da aprendizagem corporativa em teste de software considerando as publicações, dos últimos 5 anos, em tal campo de pesquisa. Até o momento, não se faz presente na literatura estudos que proporcionem a aprendizagem corporativa em teste de software.

Dentre os 76 estudos analisados, foram encontrados 4 artigos que abordam a GC e a ontologia integrada como estratégias educacionais de aprendizagem corporativo. Entretanto, as propostas apresentadas nesses trabalhos demandam um alto investimento por parte das empresas e também, um certo nível de maturidade para que a GC ou a ontologia integrada sejam implementadas. 
VIII Congresso Brasileiro de Informática na Educação (CBIE 2019)

Anais do XXV Workshop de Informática na Escola (WIE 2019)

Dada a complexidade de aplicação de ambas e a relevância de melhorar a qualidade dos produtos de software disponíveis no mercado, tem-se o hackathon como uma forma de possibilitar a aprendizagem corporativa em teste de software capaz de disseminar e preservar o conhecimento adquirido durante o ciclo de vida de desenvolvimento. $\mathrm{O}$ uso de hackathons são uma tendência crescente para promover o engajamento entre a equipe em atividades relacionadas à criatividade, ao design e à resolução de problemas. Dessa forma, o hackathon oferece uma excelente estrutura para que a aprendizagem corporativa em teste de software seja desenvolvida de maneira efetiva e inclusiva.

\section{Referências}

Bartié, A. (2002). Garantia de Qualidade de Software. Campus, Rio de Janeiro.

Byrne, J. R., O'Sullivan, K., and Sullivan, K. (2017). An iot and wearable technology hackathon for promoting careers in computer science. IEEE Transactions on Education, 60:50-58.

de Souza, E. F., Falbo, R. A., and Vijaykumar, N. L. (2015). Knowledge management initiatives in software testing: A mapping study. Information and Software Technology, 57:378-391.

Kitchenham, B. and Charter, S. (2007). Guidelines for performing systematic literature reviews in software engineering. Technical report, Keele University and University of Durham, United Kingdom.

Meireles, M. A. C. and Bonifácio, B. A. (2015). Uso de métodos ágeis e aprendizagem baseada em problema no ensino de engenharia de software: Um relato de experiência. Anais do XXVI Simpósio Brasileiro de Informática na Educação.

Menolli, A., Reinehr, S., and Malucelli, A. (2013). Organizational learning applied to software engineering: A systematic review. International Journal of Software Engineering and Knowledge Engineering, 23:1153-1175.

Okoli, C. and Schabram, K. (2010). A guide to conducting a systematic literature review of information systems research. Sprouts: Working Papers on Information Systems, pages $10-26$.

Shongwe, M. M. (2017). Knowledge management in small software development organisations: A south african perspective. South African Journal of Information Management, 19:1-9.

Staab, T. C. (2003). Improving the test process: Looking at the test process - getting started. The Journal of the Software Testing Professionals.

Valaski, J., Reinehr, S., and Malucelli, A. (2017). An ontology to support the classification of learning material in an organizational learning environment: An evaluation. Interactive Technology and Smart Education, 14:67-87. 